\title{
AN ITERATIVE METHOD FOR MULTIPLE STOPPING: CONVERGENCE AND STABILITY
}

\author{
CHRISTIAN BENDER *** AND \\ JOHN SCHOENMAKERS, ${ }^{*}$ Weierstrass Institute for Applied Analysis and Stochastics
}

\begin{abstract}
We present a new iterative procedure for solving the multiple stopping problem in discrete time and discuss the stability of the algorithm. The algorithm produces monotonically increasing approximations of the Snell envelope which coincide with the Snell envelope after finitely many steps. Unlike backward dynamic programming, the algorithm allows us to calculate approximative solutions with only a few nestings of conditional expectations and is, therefore, tailor-made for a plain Monte Carlo implementation.
\end{abstract}

Keywords: Optimal stopping; policy improvement; multiple callable financial derivatives

2000 Mathematics Subject Classification: Primary 60G40; 62L15; 91B28

\section{Introduction}

Financial derivatives with several early exercise rights play an important role in different markets, e.g. in electricity markets (swing options) and interest rate markets (chooser flexible caps). The pricing problem for such instruments is equivalent to a multiple stopping problem which is usually solved in practice using trinomial forests; see Jaillet $e t$ al. (2004) and the references therein. However, this pricing procedure is restricted to models for low-dimensional underlying processes, as trees tend to explode as the dimension of the underlying process increases (the so-called curse of dimensionality).

Obviously, multiple callable instruments with respect to a high-dimensional interest rate model such as the popular LIBOR market model and multiple callable options on a basket of several assets do not meet this restriction. New pricing methods for instruments with early exercise opportunities, based on high-dimensional underlying processes, are therefore called for.

Only in recent years have several approaches been proposed to overcome the curse of dimensionality for American-style derivatives, that is, in the case of a single exercise right. These methods basically rely on Monte Carlo simulation and can be roughly divided into three groups. The first group directly employs a recursive scheme for solving the stopping problem, known as backward dynamic programming. Different techniques are applied to approximate the nested conditional expectations. The stochastic mesh method of Broadie and Glasserman (2004) and Broadie et al. (2000) and the least-squares regression method of Longstaff and Schwartz (2001) are among the most popular approaches in this group. An alternative to backward dynamic programming is to approximate the exercise boundary by simulation; see,

Received 5 April 2005; revision received 21 March 2006.

* Postal address: Weierstrass Institute for Applied Analysis and Stochastics, Mohrenstr. 39, D-10117 Berlin, Germany.

** Email address: bender@wias-berlin.de

Supported by the DFG Research Center MATHEON, Berlin. 
e.g. Andersen (1999), Ibáñez and Zapatero (2004), and Milstein et al. (2004). The third group relies on a dual approach developed by Rogers (2002), Haugh and Kogan (2004), and, in a multiplicative setting, by Jamshidian (2006). By duality, tight upper bounds may be constructed from given approximative processes.

The methods in these three categories can be transferred from applying to one exercise opportunity to applying to several, because the multiple stopping problem is equivalent to a system of nested single stopping problems. Meinshausen and Hambly (2004) suggested an extension to several exercise rights of the Longstaff and Schwartz (2001) algorithm along these lines. Their main contribution was a derivation of the dual formulation under several exercise rights. Ibáñez (2004) presented a generalization to multiple exercise opportunities of the results of Ibáñez and Zapatero (2004).

The results of the present paper are twofold. First, we suggest an algorithm for the multiple stopping problem which generalizes a procedure recently introduced by Kolodko and Schoenmakers (2006) for the single stopping problem. Second, we analyze stability of the algorithm under one exercise right as well as under several.

Policy-improvement algorithms, such as the one proposed by Kolodko and Schoenmakers (2006), address one of the main drawbacks of the backward dynamic programming scheme. Suppose that exercise can take place at one of $k$ time instances. Then, in order to obtain the value of the optimal stopping problem via backward dynamic programming, one has to calculate nested conditional expectations of order $k$. No approximation of the value at time 0 is available prior to the evaluation of the $k$ th nested conditional expectations. This prevents the use of plain Monte Carlo simulations in approximating the conditional expectations and necessitates the use of more complicated approximation procedures for these quantities. Contrarily, the algorithm of Kolodko and Schoenmakers (2006) yields approximations of the value function at time 0 at every iteration step, which monotonically increase to coincide with the Snell envelope. This allows one to calculate approximations of the Snell envelope via a plain Monte Carlo simulation, if the underlying process is Markovian. Indeed, it was shown in Kolodko and Schoenmakers (2006) that good approximations can be obtained even for very high-dimensional $(d=40$ !) problems.

In fact, the main advantage of the algorithm of Kolodko and Schoenmakers (2006) would be lost if a multiexercise version were straightforwardly defined as a nesting of one-exercise versions. This would produce nested conditional expectations at each iteration step and, thus, again prevent the use of a plain Monte Carlo implementation. Instead, we present a multiple exercise version of the policy-improvement algorithm in such a way that the order of nestings does not depend on the number of exercise rights. It is therefore tailored for plain Monte Carlo simulation of the conditional expectations. We also prove that the algorithm coincides with the Snell envelope under $L$ exercise rights after the same number of iterations as are needed for the nested dynamic programming algorithm proposed in Carmona and Touzi (2006). This shows that our algorithm is theoretically as good as backward dynamic programming, and may be superior from a practical point of view.

The second contribution of our paper is a stability analysis for the policy-improvement algorithm of Kolodko and Schoenmakers (2006) and its multiexercise extension. In the case of a single exercise right, the stability result can be put into words as follows (recall that one can think of the stopping problem as that of an investor trying to maximize his expected gain). The shortfall of the investor's expected gain corresponding to $m$ steps of the perturbed algorithm below the expected gain corresponding to $m$ steps of the theoretical algorithm converges to 0 . Surprisingly, it can happen that the perturbed algorithm performs better than the theoretical one 
(as is shown in Example 4.1, below). A similar result is obtained in the multiexercise case. This stability analysis provides a rigorous basis for a Monte Carlo implementation of the algorithms.

Finally, we note that standard policy iterations for dynamic programming (see, e.g. Puterman (1994), Kushner and Dupuis (2001), and Bertsekas (2001)) are based on lower approximations of the continuation value due to a (suboptimal) strategy. In contrast, the multiple stopping problem involves a lower approximation of the continuation value and the cash flow, and, hence, a lower approximation on both sides of the inequality in the exercise criterion. The approximation of the cash flow is crucial to avoid additional nestings of conditional expectations and, therefore, reduces the computational complexity. In fact, in our algorithm both lower approximations are calculated by means of the same (suboptimal) strategy and the monotone improvement property is nonetheless established.

The paper is organized as follows. In Section 2 we pose the multiple stopping problem and explain its connection to the single stopping problem. Then, in Section 3, we state the multiple exercise algorithm and prove its convergence. In particular, in Subsections 3.2 and 3.3 we put our main emphasis on the analysis of the building blocks of the algorithm, called one-step improvements. The results of Subsections 3.2 and 3.3 are crucial for the discussion of stability in Section 4.

\section{On the multiple stopping problem}

Suppose that $(Z(i): i=0,1, \ldots, k)$ is a nonnegative stochastic process in discrete time on a probability space $(\Omega, \mathcal{F}, \mathrm{P})$ adapted to some filtration $\left(\mathcal{F}_{i}: 0 \leq i \leq k\right)$ which satisfies

$$
\sum_{i=1}^{k} \mathrm{E}[|Z(i)|]<\infty
$$

We may think of the process $Z$ as a cash flow which an investor may exercise $L$ times. The investor's problem is to maximize his expected gain by exercising optimally. He is subjected to the additional constraint that he has to wait a minimal time $\delta \in \mathbb{N}$ between exercising two rights. The introduction of $\delta$ avoids mathematical trivialities, as without it the investor could exercise all rights at the same time. To emphasize that the introduction of $\delta$ is not a mathematical oddity, we will refer to $\delta$ as the refracting period, following the terminology of swing options.

We now formalize the multiple stopping problem. For notational convenience we trivially extend the cash flow process by defining $Z(i)=0$ and $\mathscr{F}_{i}=\mathscr{F}_{k}$, for $i>k$. Let us define $\delta_{i}(L, \delta)$ as the set of $\mathscr{F}_{i}$ stopping vectors $\left(\tau_{1}(i), \ldots, \tau_{L}(i)\right)$ such that $i \leq \tau_{1}(i)$ and $\tau_{j-1}(i)+\delta \leq \tau_{j}(i)$ for all $j, 2 \leq j \leq L$. The multiple stopping problem may then be stated as follows: find a family of stopping vectors $\tau^{*}(i) \in \wp_{i}(L, \delta)$ such that, for $0 \leq i \leq k$,

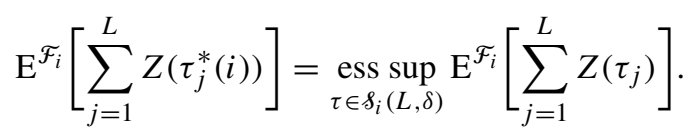

The process on the right-hand side is called the Snell envelope of $Z$ under $L$ exercise rights and we denote it by $Y_{L}^{*}(i)$. We sometimes write $Y^{*}(i)$ for $Y_{1}^{*}(i)$.

The case of one exercise right, $L=1$, is very well studied. We here collect the following facts, which can be found in Neveu (1975, pp. 122-124).

1. The Snell envelope, $Y^{*}$, of $Z$ under one exercise right is the smallest supermartingale that dominates $Z$. 
2. A family of optimal stopping times for the stopping problem with one exercise right is given by

$$
\tau^{*}(i)=\inf \left\{j: j \geq i, Z(j) \geq \mathrm{E}^{\mathcal{F}_{j}}\left[Y^{*}(j+1)\right]\right\}, \quad 0 \leq i \leq k .
$$

If several optimal stopping families exist, then the above family is the family of smallest optimal stopping times.

The multiple stopping problem can be reduced to $L$ nested stopping problems with one exercise right. We briefly explain the reduction.

Define a sequence of processes $\left(X_{0}, \ldots, X_{L}, \ldots\right)$ as follows: $X_{0}:=0, X_{1}:=Y_{1}^{*}$ is the Snell envelope of $Z$, and $X_{L}, L \geq 2$, is the Snell envelope of the cash flow $Z(i)+\mathrm{E}^{\mathcal{F}_{i}}\left[X_{L-1}(i+\delta)\right]$ under one exercise right. For $L=1,2, \ldots$, we also define

$$
\sigma_{L}^{*}(i)=\inf \left\{j: j \geq i, Z(j)+\mathrm{E}^{\mathcal{F}_{j}}\left[X_{L-1}(j+\delta)\right] \geq \mathrm{E}^{\mathcal{F}_{j}}\left[X_{L}(j+1)\right]\right\}, \quad i \geq 0,
$$

i.e. the smallest optimal stopping families for the sequence of single stopping problems. It is straightforward to show, by induction on $L$, that

$$
Y_{L}^{*}(i)=X_{L}(i), \quad 1 \leq i \leq k,
$$

and that a family of optimal stopping vectors for the multiple stopping problem with $L$ exercise rights and cash flow $Z$ is given by

$$
\begin{aligned}
\tau_{1, L}^{*}(i) & =\sigma_{L}^{*}(i), \\
\tau_{d+1, L}^{*}(i) & =\tau_{d, L-1}^{*}\left(\sigma_{L}^{*}(i)+\delta\right), \quad 1 \leq d \leq L-1 .
\end{aligned}
$$

Note that, due to the convention that $Z(i)=0$ for $i>k$, we have $\tau_{1, L}^{*}(i)=\sigma_{L}^{*}(i)=i$ for $i \geq k$.

By the above reduction, any algorithm for single optimal stopping problems can, in principle, be applied iteratively to the multiple stopping problem. For example, Carmona and Touzi (2006) suggested applying backward dynamic programming iteratively to the $L$ stopping problems. However, this approach leads to high nestings of conditional expectations and, as a consequence, to tremendous simulation costs in a plain Monte Carlo approach.

\section{An algorithm for multiple stopping}

\subsection{The algorithm}

We are now going to present an algorithm which simultaneously improves the Snell envelope under $L=1, \ldots, D$ exercise rights, with the order of nested conditional expectations for a given number of iterations independent of $L$. In the case of a single exercise right it coincides with the procedure of Kolodko and Schoenmakers (2006). The building block of the algorithm is, as in the case of one exercise right, a policy improvement. More precisely, suppose that we are given the families of stopping times $\sigma_{L}(i), 0 \leq i \leq k, 1 \leq L \leq D$, trivially extended by defining $\sigma_{L}(i)=i$ for $i>k$. Recall that $k$ is the time horizon of the real cash flow process. We are interested in the Snell envelope with $L, 1 \leq L \leq D$, exercise rights and refracting period $\delta$. We interpret $\sigma_{L}(i)$ as the time at which the investor exercises (possibly in a suboptimal way) the first of his $L$ rights, given that he has not exercised any prior to time $i$. This interpretation requires that the stopping families $\sigma_{L}$ under consideration be consistent in the sense of the following definition. 
Definition 3.1. A family of integer-valued stopping times $(\tau(i): 0 \leq i \leq k)$ is said to be consistent if, for $0 \leq i<k$,

$$
\begin{gathered}
i \leq \tau(i) \leq k, \quad \tau(k):=k, \\
\tau(i)>i \quad \Longrightarrow \quad \tau(i)=\tau(i+1) .
\end{gathered}
$$

Given consistent stopping families $\sigma_{L}, L=1,2, \ldots$, we define associated stopping families $\tau_{d+1, L}$ via

$$
\begin{aligned}
\tau_{1, L}(i) & =\sigma_{L}(i), \\
\tau_{d+1, L}(i) & =\tau_{d, L-1}\left(\sigma_{L}(i)+\delta\right), \quad 1 \leq d \leq L-1 .
\end{aligned}
$$

We can interpret $\tau_{d, L}(i)$ as the time at which the investor exercises the $d$ th of his $L$ exercise rights, provided that he has not exercised his first right prior to time $i$. An approximation of the Snell envelope with $L$ exercise rights is now given by

$$
Y_{L}\left(i ; \sigma_{1}, \ldots, \sigma_{L}\right):=\mathrm{E}^{\mathcal{F}_{i}}\left[\sum_{d=1}^{L} Z\left(\tau_{d, L}(i)\right)\right] .
$$

Note that $Y_{L}\left(i ; \sigma_{1}, \ldots, \sigma_{L}\right)$ has a simple interpretation as the expected gain (conditional on $\mathcal{F}_{i}$ ) the investor obtains when he employs the stopping families $\sigma_{1}, \ldots, \sigma_{L}$ in exercising the cash flows. We then introduce intermediate processes,

$$
\hat{Y}_{L}\left(i ; \sigma_{1}, \ldots, \sigma_{L}\right):=\max _{i+1 \leq p \leq k} \mathrm{E}^{\mathcal{F}_{i}}\left[\sum_{d=1}^{L} Z\left(\tau_{d, L}(p)\right)\right],
$$

on which a further exercise criterion,

$$
\tilde{\sigma}_{L}(i):=\inf \left\{j: j \geq i, Z(j)+\mathrm{E}^{\mathcal{F}_{j}}\left[Y_{L-1}\left(j+\delta ; \sigma_{1}, \ldots, \sigma_{L-1}\right)\right] \geq \hat{Y}_{L}\left(j ; \sigma_{1}, \ldots, \sigma_{L}\right)\right\},
$$

is built with $Y_{0}(i):=0$. Note that $\tilde{\sigma}_{L}(k)=k$ since $\max \varnothing=-\infty$ and, obviously, that the stopping families $\tilde{\sigma}_{L}$ are consistent for $1 \leq L \leq D$.

Given consistent starting families of stopping times $\sigma_{L}^{(0)}, 1 \leq L \leq D$, we iteratively define

$$
\begin{aligned}
\sigma_{L}^{(m)}(i) & :=\tilde{\sigma}_{L}^{(m-1)}(i), \\
Y_{L}^{(m)}(i) & :=Y_{L}\left(i ; \sigma_{1}^{(m)}, \ldots, \sigma_{L}^{(m)}\right) .
\end{aligned}
$$

Here $\tilde{\sigma}_{L}^{(\cdot)}$ is the result of applying the tilde operation to $\sigma_{L}^{(\cdot)}$. Similar notation is used below. Canonical consistent starting families are given, for instance, by $\sigma_{L}^{(0)}(i)=i, L=1,2, \ldots$.

Theorem 3.1. Suppose that the stopping families $\sigma_{L}^{(0)}(i)$ are consistent for all $L, 1 \leq L \leq D$. Then, for all $m \in \mathbb{N}, L, 1 \leq L \leq D$, and $i, 0 \leq i \leq k$,

$$
Y_{L}^{(m+1)}(i) \geq Y_{L}^{(m)}(i) .
$$

Moreover, for $m \geq k-i$,

$$
Y_{L}^{(m)}(i)=Y_{L}^{*}(i)
$$

where $Y_{L}^{*}$ denotes the Snell envelope of $Z$ under $L$ exercise rights. 
The dynamic programming scheme suggests defining another approximation of the Snell envelope, namely

$$
\begin{aligned}
y_{L}\left(i ; \sigma_{1}, \ldots, \sigma_{L}\right):=\max \left\{Z(i)+\mathrm{E}^{\mathcal{F}_{j}}\left[Y_{L-1}\left(i+\delta ; \sigma_{1}, \ldots, \sigma_{L-1}\right)\right],\right. \\
\left.\quad \mathrm{E}^{\mathcal{F}_{i}}\left[Y_{L}\left(i+1 ; \sigma_{1}, \ldots, \sigma_{L}\right)\right]\right\}, \quad 1 \leq L \leq D, 0 \leq i \leq k,
\end{aligned}
$$

given consistent stopping families $\sigma_{1}, \ldots, \sigma_{D}$. Based on $y_{L}$, we will also consider the modified algorithm

$$
\begin{aligned}
\sigma_{L}^{(m)}(i) & :=\tilde{\sigma}_{L}^{(m-1)}(i), \\
y_{L}^{(m)}(i) & :=y_{L}\left(i ; \sigma_{1}^{(m)}, \ldots, \sigma_{L}^{(m)}\right),
\end{aligned}
$$

with the same stopping families $\sigma_{L}^{(m)}$ as in (3.6). The approximation $y_{L}$ does not admit as intuitive an interpretation as does $Y_{L}$, but the modified algorithm yields better approximations than the one based on $Y_{L}$.

Theorem 3.2. All assertions of Theorem 3.1 hold with $Y_{L}^{(m)}$ replaced by $y_{L}^{(m)}$. Moreover, for all $m \in \mathbb{N}, L, 1 \leq L \leq D$ and $i, 0 \leq i \leq k$,

$$
y_{L}^{(m)}(i) \geq Y_{L}^{(m)}(i)
$$

(provided that both algorithms are initiated with the same stopping families).

We prove Theorems 3.1 and 3.2 in Subsection 3.4. Before we scrutinize the building blocks of the algorithm, which we will refer to as one-step improvements in the following subsections, let us briefly discuss the implementation of the algorithm.

3.1.1. On the implementation of the algorithm. Although Theorems 3.1 and 3.2 state convergence to the Snell envelope in finitely many steps, in practice only a few steps (not the whole algorithm) may be calculated. In this respect the main benefit of the theorems is that every step improves upon the previous one. Specifically, we recommend the following procedure, if the underlying process has a Markovian structure.

1. Choose appropriate starting families. If no better choice is known a priori, start with the canonical families $\sigma_{L}^{(0)}(i)=i$.

2. Apply the plain Monte Carlo estimator to approximate all (conditional) expectations in the iteration steps based on (3.3) and (3.5), which are not known analytically.

3. The obtained approximations are (up to simulation error) lower bounds of the Snell envelope. Upper bounds (up to simulation error) can be calculated from the lower bounds by the dual method of Meinshausen and Hambly (2004).

More detailed information on the implementation, including a generalization of the pseudocode of Schoenmakers (2005, pp. 166ff.) to the case of two exercise rights, can be found in an addendum provided online (Bender and Schoenmakers (2006)). Simulation results in the case of a single exercise right were presented in Kolodko and Schoenmakers (2006) for a Bermudan swaption on a 40-dimensional underlying.

\subsection{A generalization of the one-step improvement in the case of one exercise right}

We now investigate a single improvement step in the case of one exercise right and generalize results of Kolodko and Schoenmakers (2006). These generalizations will be of crucial importance in investigating the stability of the proposed algorithm in Section 4. 
Suppose that a consistent stopping family $(\tau(i): 1 \leq i \leq k)$ is given. We then define the process

$$
Y(i ; \tau):=\mathrm{E}^{\mathcal{F}_{i}}[Z(\tau(i))] .
$$

Based on the sequence $(\tau(i): 1 \leq i \leq k)$, Kolodko and Schoenmakers (2006) constructed a new family, $(\tilde{\tau}(i): 1 \leq i \leq k)$, in the following way. Introduce an intermediate process

$$
\tilde{Y}(i ; \tau):=\max _{\{p: i \leq p \leq k\}} \mathrm{E}^{\mathcal{F}_{i}}[Z(\tau(p))]
$$

which serves as a new exercise criterion, i.e.

$$
\begin{aligned}
\tilde{\tau}(i) & :=\inf \{j: i \leq j \leq k, \tilde{Y}(j ; \tau) \leq Z(j)\} \\
& =\inf \left\{j: i \leq j \leq k, \max _{\{p: j \leq p \leq k\}} \mathrm{E}^{\mathcal{F}_{j}}[Z(\tau(p))] \leq Z(j)\right\}, \quad 0 \leq i \leq k .
\end{aligned}
$$

Kolodko and Schoenmakers (2006, Theorem 3.1) showed that $\tilde{\tau}$ is an improvement on $\tau$ in the sense that the new strategy promises a higher expected gain for the investor than does the old one, i.e. $Y(i ; \tilde{\tau}) \geq \tilde{Y}(i ; \tau) \geq Y(i ; \tau)$.

Our first aim is to extend this chain of inequalities to a wider class of stopping families than $\tilde{\tau}$. To this end we first compare the intermediate processes $\tilde{Y}(i ; \tau)$ and

$$
\hat{Y}(i ; \tau):=\max _{\{p: i+1 \leq p \leq k\}} \mathrm{E}^{\mathcal{F}_{i}}[Z(\tau(p))] .
$$

By $\mathbf{1}_{A}$ we denote the indicator function of the set $A$.

Lemma 3.1. Suppose the stopping family $\tau$ is consistent. Then, for $0 \leq i \leq k$,

$$
\tilde{Y}(i ; \tau)=\mathbf{1}_{\{\tau(i)>i\}} \hat{Y}(i ; \tau)+\mathbf{1}_{\{\tau(i)=i\}} \max \{\hat{Y}(i ; \tau), Z(i)\} .
$$

In particular,

$$
Z(i) \geq \tilde{Y}(i ; \tau) \quad \Longleftrightarrow \quad Z(i) \geq \hat{Y}(i ; \tau)
$$

and

$$
\tilde{\tau}(i)=\inf \{j: i \leq j \leq k, \hat{Y}(j) \leq Z(j)\} .
$$

Proof. By property (3.1), we have

$$
\begin{aligned}
\mathrm{E}^{\mathcal{F}_{i}}[Z(\tau(i))] & =\mathrm{E}^{\mathcal{F}_{i}}\left[\mathbf{1}_{\{\tau(i)=i\}} Z(i)\right]+\mathrm{E}^{\mathcal{F}_{i}}\left[\mathbf{1}_{\{\tau(i)>i\}} Z(\tau(i+1))\right] \\
& =\mathbf{1}_{\{\tau(i)=i\}} Z(i)+\mathbf{1}_{\{\tau(i)>i\}} \mathrm{E}^{\mathcal{F}_{i}}[Z(\tau(i+1))] .
\end{aligned}
$$

Since

$$
\tilde{Y}(i ; \tau)=\max \left\{\hat{Y}(i ; \tau), \mathrm{E}^{\mathcal{F}_{i}}[Z(\tau(i))]\right\},
$$

(3.9) follows with (3.10) and (3.11) as immediate consequences.

We next define another stopping family, namely

$$
\hat{\tau}(i):=\inf \{j: i \leq j \leq k, \hat{Y}(j)<Z(j)\} .
$$

Clearly, by (3.11),

$$
\hat{\tau}(i) \geq \tilde{\tau}(i)
$$


Theorem 3.3. Let $(\tau(i): 1 \leq i \leq k)$ be a consistent stopping family. Suppose that $(\bar{\tau}(i): 1 \leq$ $i \leq k)$ is also consistent and satisfies

$$
\tilde{\tau}(i) \leq \bar{\tau}(i) \leq \hat{\tau}(i), \quad 0 \leq i \leq k .
$$

Then

$$
Y(i ; \bar{\tau}) \geq \tilde{Y}(i ; \tau) \geq Y(i ; \tau), \quad 0 \leq i \leq k .
$$

Remark 3.1. Obviously, the choices $\bar{\tau}=\tilde{\tau}$ and $\bar{\tau}=\hat{\tau}$ are examples of a family $\bar{\tau}$ satisfying (3.1) and (3.12).

Proof of Theorem 3.3. The right-hand inequality is trivial. We prove the left-hand inequality by backward induction on $i$. For $i=k$, note that $Y(k ; \bar{\tau})=Z(k)=\tilde{Y}(k ; \tau)$. Now suppose that $0 \leq i \leq k-1$ and that the assertion holds for $i+1$. It holds that $\{\bar{\tau}(i)=i\} \subset\{\tilde{\tau}(i)=i\}$, by (3.12). Hence, on the set $\{\bar{\tau}(i)=i\}$, we obtain

$$
Y(i ; \bar{\tau})=Z(i) \geq \tilde{Y}(i ; \tau) .
$$

However, on $\{\bar{\tau}(i)>i\}$ we have $\bar{\tau}(i)=\bar{\tau}(i+1)$ and the induction hypothesis thus yields

$$
\begin{aligned}
Y(i ; \bar{\tau}) & =\mathrm{E}^{\mathcal{F}_{i}}[Z(\bar{\tau}(i+1))]=\mathrm{E}^{\mathcal{F}_{i}}[Y(i+1 ; \bar{\tau})] \geq \mathrm{E}^{\mathcal{F}_{i}}[\tilde{Y}(i+1 ; \tau)] \\
& =\mathrm{E}^{\mathcal{F}_{i}}\left[\max _{i+1 \leq p \leq k} \mathrm{E}^{\mathcal{F}_{i+1}}[Z(\tau(p))]\right] \geq \max _{i+1 \leq p \leq k} \mathrm{E}^{\mathcal{F}_{i}}[Z(\tau(p))] \\
& =\hat{Y}(i ; \tau) .
\end{aligned}
$$

Property (3.12) implies that $\{\bar{\tau}(i)>i\} \subset\{\hat{\tau}(i)>i\}$. Thus, on $\{\bar{\tau}(i)>i\}, \hat{Y}(i ; \tau) \geq Z(i)$ and, by (3.9), $\hat{Y}(i ; \tau)=\tilde{Y}(i ; \tau)$.

Motivated by the previous theorem we introduce the notion of an improver.

Definition 3.2. Suppose that $\tau$ is a consistent stopping family. A stopping family $\bar{\tau}$ is called an improver of $\tau$ if it is consistent and satisfies (3.12) for $0 \leq i \leq k$.

The next theorem provides another justification for the name 'improver'.

Theorem 3.4. Suppose that $\tau$ is a consistent stopping family and that $\bar{\tau}$ is an improver of $\tau$. Then

$$
Y(i ; \tau)=Y^{*}(i), \quad i \geq j+1
$$

implies that

$$
Y(i ; \bar{\tau})=Y^{*}(i), \quad i \geq j .
$$

Proof. We will exploit the fact that the Snell envelope is the smallest supermartingale dominating $Z$. By Theorem 3.3, for $0 \leq i \leq k-1$ we have

$$
Y(i ; \bar{\tau}) \geq \tilde{Y}(i ; \tau) \geq \mathrm{E}^{\mathcal{F}_{i}}[Z(\tau(i+1))]=\mathrm{E}^{\mathcal{F}_{i}}[Y(i+1 ; \tau)] .
$$

Therefore, for $j \leq i \leq k-1$,

$$
Y(i ; \bar{\tau}) \geq \mathrm{E}^{\mathcal{F}_{i}}\left[Y^{*}(i+1)\right] \geq \mathrm{E}^{\mathcal{F}_{i}}[Y(i+1 ; \bar{\tau})] .
$$

This means that $(Y(i ; \bar{\tau}): j \leq i \leq k)$ is a supermartingale. We may also deduce from Theorem 3.3 that, for $0 \leq i \leq k$,

$$
Y(i ; \bar{\tau}) \geq \mathbf{1}_{\{\bar{\tau}(i)=i\}} Z(i)+\mathbf{1}_{\{\bar{\tau}(i)>i\}} \tilde{Y}(i ; \tau) .
$$


However, as in the proof of Theorem 3.3, we obtain

$$
\mathbf{1}_{\{\bar{\tau}(i)>i\}} \tilde{Y}(i ; \tau) \geq \mathbf{1}_{\{\bar{\tau}(i)>i\}} \hat{Y}(i ; \tau) \geq \mathbf{1}_{\{\bar{\tau}(i)>i\}} Z(i) .
$$

Thus, $(Y(i ; \bar{\tau}): j \leq i \leq k)$ is a supermartingale dominating $Z$. Therefore,

$$
Y(i ; \bar{\tau}) \geq Y^{*}(i) \text { for } i \geq j
$$

Proving the reverse inequality is trivial.

Remark 3.2. The proof of the previous theorem shows that, for any improver $\bar{\tau}$,

$$
Y(i ; \bar{\tau}) \geq Z(i), \quad 0 \leq i \leq k .
$$

We end this section with a comparison between different improvers.

Proposition 3.1. Suppose that $\tau$ is consistent and that $\bar{\tau}$ is an improver of $\tau$. Then, for all $0 \leq i \leq k$,

$$
Y(i ; \hat{\tau}) \geq Y(i ; \bar{\tau}) \geq Y(i ; \tilde{\tau}) .
$$

Proof. We prove only the right-hand inequality; the proof of the left-hand inequality is similar. For $i=k$ equality holds. Suppose that $0 \leq i \leq k-1$ and that the inequality holds for $i+1$. Then, on $\{\bar{\tau}(i)>i\} \cap\{\tilde{\tau}(i)>i\}$,

$$
Y(i ; \bar{\tau})=\mathrm{E}^{\mathcal{F}_{i}}[Y(i+1 ; \bar{\tau})] \geq \mathrm{E}^{\mathcal{F}_{i}}[Y(i+1 ; \tilde{\tau})]=Y(i ; \tilde{\tau})
$$

by the induction hypothesis. On $\{\bar{\tau}(i)>i\} \cap\{\tilde{\tau}(i)=i\}$ we have

$$
Y(i ; \bar{\tau}) \geq Z(i)=Y(i ; \tilde{\tau})
$$

by (3.13). Finally, the set $\{\bar{\tau}(i)=i\} \cap\{\tilde{\tau}(i)>i\}$ is evanescent by the definition of an improver.

\subsection{The one-step improvement in the case of several exercise rights}

We now investigate the one-step improvement under several exercise rights defined in (3.2)-(3.5). To this end, suppose that consistent stopping families $\sigma_{1}, \ldots, \sigma_{D}$ are given. The following obvious representations of $Y_{L}\left(i ; \sigma_{1}, \ldots, \sigma_{L}\right)$ and $\hat{Y}_{L}\left(i ; \sigma_{1}, \ldots, \sigma_{L}\right)$ allow us to extend Theorem 3.3 to the case of several exercise rights.

Lemma 3.2. For $2 \leq L \leq D$ and $0 \leq i \leq k$, define

$$
Z_{L}\left(i ; \sigma_{1}, \ldots, \sigma_{L-1}\right)=Z(i)+\mathrm{E}^{\mathcal{F}_{i}}\left[Y_{L-1}\left(i+\delta ; \sigma_{1}, \ldots, \sigma_{L-1}\right)\right] .
$$

Then

$$
\begin{aligned}
Y_{L}\left(i ; \sigma_{1}, \ldots, \sigma_{L}\right) & =\mathrm{E}^{\mathcal{F}_{i}}\left[Z_{L}\left(\sigma_{L}(i) ; \sigma_{1}, \ldots, \sigma_{L-1}\right)\right], \\
\hat{Y}_{L}\left(i ; \sigma_{1}, \ldots, \sigma_{L}\right) & =\max _{i+1 \leq p \leq k} \mathrm{E}^{\mathcal{F}_{i}}\left[Z_{L}\left(\sigma_{L}(p) ; \sigma_{1}, \ldots, \sigma_{L-1}\right)\right] .
\end{aligned}
$$

By the previous lemma, we may rewrite $\tilde{\sigma}_{L}$ (see (3.5)) as

$$
\tilde{\sigma}_{L}(i)=\inf \left\{j: j \geq i, Z_{L}\left(j ; \sigma_{1}, \ldots, \sigma_{L-1}\right) \geq \max _{p \geq j+1} E^{\mathcal{F}_{j}}\left[Z_{L}\left(\sigma_{L}(p) ; \sigma_{1}, \ldots, \sigma_{L-1}\right)\right]\right\},
$$

for $0 \leq i \leq k$. Consequently, the step from $\sigma_{L}$ to $\tilde{\sigma}_{L}$ is a one-step improvement with one exercise right and cash flow $Z_{L}\left(\cdot ; \sigma_{1}, \ldots, \sigma_{L-1}\right)$.

As in the case of one exercise right, we also consider the stopping family

$$
\hat{\sigma}_{L}(i)=\inf \left\{j: j \geq i, Z_{L}\left(j ; \sigma_{1}, \ldots, \sigma_{L-1}\right)>\max _{p \geq j+1} \mathrm{E}^{\mathcal{F}_{j}}\left[Z_{L}\left(\sigma_{L}(p) ; \sigma_{1}, \ldots, \sigma_{L-1}\right)\right]\right\},
$$

for $0 \leq i \leq k$. 
Definition 3.3. A stopping family $\bar{\sigma}_{L}$ is said to be an L-improver of $\sigma_{L}$ with respect to $\left(\sigma_{1}, \ldots, \sigma_{L-1}\right)$ if $\bar{\sigma}_{L}$ is consistent and

$$
\tilde{\sigma}_{L}(i) \leq \bar{\sigma}_{L}(i) \leq \hat{\sigma}_{L}(i)
$$

In an abuse of this terminology, we will simply speak of an improver when $L$ and $\left(\sigma_{1}, \ldots, \sigma_{L-1}\right)$ are evident from the context.

We now state a generalization of Theorem 3.3 which justifies the name 'improver' here.

Theorem 3.5. Suppose that consistent stopping families $\sigma_{1}, \ldots, \sigma_{D}$ with respective improvers $\bar{\sigma}_{1}, \ldots, \bar{\sigma}_{D}$ are given. Then, for $1 \leq L \leq D$, the following chain of inequalities holds:

$$
\begin{aligned}
Y_{L}\left(i ; \bar{\sigma}_{1}, \ldots, \bar{\sigma}_{L}\right) & \geq Y_{L}\left(i ; \sigma_{1}, \ldots, \sigma_{L-1}, \bar{\sigma}_{L}\right) \\
& \geq Y_{L}\left(i ; \sigma_{1}, \ldots, \sigma_{L-1}, \tilde{\sigma}_{L}\right) \\
& \geq \max \left\{Y_{L}\left(i ; \sigma_{1}, \ldots, \sigma_{L}\right), \hat{Y}_{L}\left(i ; \sigma_{1}, \ldots, \sigma_{L}\right)\right\} .
\end{aligned}
$$

Proof. By the previous considerations, $\bar{\sigma}_{L}$ is also a 1-improver of $\sigma_{L}$ with respect to the cash flow $Z_{L}\left(\cdot ; \sigma_{1}, \ldots, \sigma_{L-1}\right)$ (with the convention that $Z_{1}=Z$ ). In view of Lemma 3.2, the second inequality follows from Proposition 3.1 and the third from Theorem 3.3. We will prove the first inequality by induction on $L$. Note that the inequality is trivial for $L=1$. The step from $L-1$ to $L$ can be shown as follows. By Lemma 3.2, we have

$$
\begin{aligned}
& Y_{L}\left(i ; \bar{\sigma}_{1}, \ldots, \bar{\sigma}_{L}\right)-Y_{L}\left(i ; \sigma_{1}, \ldots, \sigma_{L-1}, \bar{\sigma}_{L}\right) \\
&=\mathrm{E}^{\mathcal{F}_{i}}\left[Z\left(\bar{\sigma}_{L}(i)\right)+Y_{L-1}\left(\bar{\sigma}_{L}(i)+\delta ; \bar{\sigma}_{1}, \ldots, \bar{\sigma}_{L-1}\right)\right] \\
&-\mathrm{E}^{\mathcal{F}_{i}}\left[Z\left(\bar{\sigma}_{L}(i)\right)+Y_{L-1}\left(\bar{\sigma}_{L}(i)+\delta ; \sigma_{1}, \ldots, \sigma_{L-1}\right)\right] \\
&= \mathrm{E}^{\mathcal{F}_{i}}\left[Y_{L-1}\left(\bar{\sigma}_{L}(i)+\delta ; \bar{\sigma}_{1}, \ldots, \bar{\sigma}_{L-1}\right)-Y_{L-1}\left(\bar{\sigma}_{L}(i)+\delta ; \sigma_{1}, \ldots, \sigma_{L-1}\right)\right] .
\end{aligned}
$$

As the second and the third asserted inequalities are already proved, the induction hypothesis (for the first inequality) implies that

$$
Y_{L-1}\left(\bar{\sigma}_{L}(i)+\delta ; \bar{\sigma}_{1}, \ldots, \bar{\sigma}_{L-1}\right) \geq Y_{L-1}\left(\bar{\sigma}_{L}(i)+\delta ; \sigma_{1}, \ldots, \sigma_{L-1}\right)
$$

Thus,

$$
Y_{L}\left(i ; \bar{\sigma}_{1}, \ldots, \bar{\sigma}_{L}\right)-Y_{L}\left(i ; \sigma_{1}, \ldots, \sigma_{L-1}, \bar{\sigma}_{L}\right) \geq 0
$$

completing the proof.

The following corollary of the previous theorem follows simply from the definition of $y_{L}$ in (3.7).

Corollary 3.1. Suppose that consistent stopping families $\sigma_{1}, \ldots, \sigma_{D}$ with respective improvers $\bar{\sigma}_{1}, \ldots, \bar{\sigma}_{D}$ are given. Then the following chain of inequalities holds for $1 \leq L \leq D$ :

$$
\begin{aligned}
y_{L}\left(i ; \bar{\sigma}_{1}, \ldots, \bar{\sigma}_{L}\right) & \geq y_{L}\left(i ; \sigma_{1}, \ldots, \sigma_{L-1}, \bar{\sigma}_{L}\right) \\
& \geq y_{L}\left(i ; \sigma_{1}, \ldots, \sigma_{L-1}, \tilde{\sigma}_{L}\right) \\
& \geq y_{L}\left(i ; \sigma_{1}, \ldots, \sigma_{L}\right) .
\end{aligned}
$$

We are now ready to give the proofs of Theorems 3.1 and 3.2. 


\subsection{Proofs of Theorems 3.1 and 3.2}

Proof of Theorem 3.1. The monotonicity assertion is a direct consequence of Theorem 3.5 since, by definition,

$$
\begin{aligned}
Y_{L}^{(m)}(i) & =Y\left(i ; \sigma_{1}^{(m)}, \ldots, \sigma_{L}^{(m)}\right), \\
\sigma_{d}^{(m+1)} & =\tilde{\sigma}_{d}^{(m)}, \quad 1 \leq d \leq L .
\end{aligned}
$$

Recall that the barred quantities in Theorem 3.5 can always be replaced with their tilded counterparts according to the definition of an improver.

To prove the second assertion, we will show by backward induction on $i$ that, for $m+i \geq k$ and all $L, 1 \leq L \leq D$,

$$
\sigma_{L}^{(m)}(i)=\sigma_{L}^{*}(i)
$$

where the family of stopping families $\left(\sigma_{1}^{*}, \ldots, \sigma_{D}^{*}\right)$ is that defined in (2.1). For $i=k$ this claim is obvious. Suppose that it holds for all $j \geq i+1$. Recall, in view of $(2.3)$, that $\left(\sigma_{1}^{*}, \ldots, \sigma_{D}^{*}\right)$ induces an optimal strategy, i.e. that $Y_{L}\left(i ; \sigma_{1}^{*}, \ldots, \sigma_{L}^{*}\right)=Y_{L}^{*}(i)$. Thus, for $j \geq i+1$ and $m+i \geq k$, we have

$$
Y_{L}^{(m-1)}(j)=Y_{L}\left(j ; \sigma_{1}^{(m-1)}, \ldots, \sigma_{L}^{(m-1)}\right)=Y_{L}\left(j ; \sigma_{1}^{*}, \ldots, \sigma_{L}^{*}\right)=Y_{L}^{*}(j)
$$

and, consequently, by the supermartingale property of the Snell envelope, also

$$
\hat{Y}_{L}\left(j-1 ; \sigma_{1}^{(m-1)}, \ldots, \sigma_{L}^{(m-1)}\right)=\max _{p \geq j} \mathrm{E}^{\mathcal{F}_{j-1}}\left[Y_{L}^{(m-1)}(p)\right]=\mathrm{E}^{\mathcal{F}_{j-1}}\left[Y_{L}^{*}(j)\right] .
$$

Hence, by (2.1), (2.2), (3.5), and (3.6), for $i+m \geq k$ we have

$$
\begin{aligned}
\sigma_{L}^{(m)}(i) & =\inf \left\{j: j \geq i, Z(j)+\mathrm{E}^{\mathcal{F}_{j}}\left[Y_{L-1}\left(j+\delta ; \sigma_{1}^{(m-1)}, \ldots, \sigma_{L-1}^{(m-1)}\right)\right]\right. \\
& \left.\quad \geq \hat{Y}_{L}\left(j ; \sigma_{1}^{(m-1)}, \ldots, \sigma_{L}^{(m-1)}\right)\right\} \\
& =\inf \left\{j: j \geq i, Z(j)+\mathrm{E}^{\mathcal{F}_{j}}\left[Y_{L-1}^{*}(j+\delta)\right] \geq \mathrm{E}^{\mathcal{F}_{j}}\left[Y_{L}^{*}(j+1)\right]\right\} \\
& =\sigma_{L}^{*}(i) .
\end{aligned}
$$

Equation (3.14) now yields $Y_{L}^{(m)}(i)=Y_{L}^{*}(i)$ for $m+i \geq k$ and all $L, 1 \leq L \leq D$.

Proof of Theorem 3.2. We first show that

$$
y_{L}\left(i ; \sigma_{1}, \ldots, \sigma_{L}\right) \geq Y_{L}\left(i ; \sigma_{1}, \ldots, \sigma_{L}\right)
$$

for all $L, 1 \leq L \leq D$, and $i, 0 \leq i \leq k$, given consistent stopping families $\sigma_{1}, \ldots, \sigma_{D}$. By Lemma 3.2 and the consistency of $\sigma_{L}$, we have

$$
\begin{aligned}
Y_{L}\left(i ; \sigma_{1}, \ldots, \sigma_{L}\right)= & \mathbf{1}_{\left\{\sigma_{L}(i)=i\right\}}\left(Z(i)+\mathrm{E}^{\mathcal{F}_{i}}\left[Y_{L-1}\left(i+\delta ; \sigma_{1}, \ldots, \sigma_{L-1}\right)\right]\right) \\
& +\mathbf{1}_{\left\{\sigma_{L}(i)>i\right\}} \mathrm{E}^{\mathcal{F}_{i}}\left[Y_{L}\left(i+1 ; \sigma_{1}, \ldots, \sigma_{L}\right)\right] .
\end{aligned}
$$

This proves (3.15) and, with the choice $\left(\sigma_{1}, \ldots, \sigma_{D}\right)=\left(\sigma_{1}^{(m)}, \ldots, \sigma_{D}^{(m)}\right)$, also $y_{L}^{(m)}(i) \geq$ $Y_{L}^{(m)}(i)$. In particular, for $m \geq k-i$, application of Theorem 3.1 yields

$$
Y_{L}^{*}(i) \geq y_{L}^{(m)}(i) \geq Y_{L}^{(m)}(i)=Y_{L}^{*}(i)
$$

The monotonicity in $m$ follows directly from Corollary 3.1.

Remark 3.3. The proofs show that after any $m \geq k-i$ improvements, not only the improvements denoted using tildes, the corresponding approximations coincide with the Snell envelope under $L$ exercise rights up from time $i$ on. 


\section{Stability}

In this section we discuss the stability of the algorithms. We start with the analysis of the one-step improvement under one exercise right. Then we prove the stability of the $Y$-algorithm (i.e. (3.6)) under one exercise right. Finally, stability of the $y_{L}$-algorithm (i.e. (3.8)) for the general case is established under an additional assumption.

\subsection{Stability of the one-step improvement $(L=1)$}

Suppose that a consistent stopping family $\tau$ is given. As we cannot expect to know the conditional expectations analytically in general, but may only calculate approximations, instead of $\tilde{\tau}(i)$ we consider a sequence of stopping families

$$
\tilde{\tau}^{(N)}(i):=\inf \left\{j: i \leq j \leq k, \hat{Y}(j ; \tau)+\varepsilon^{(N)}(j) \leq Z(j)\right\}
$$

where $N \in \mathbb{N}$ and $\varepsilon^{(N)}(i)$ is a sequence of $\mathcal{F}_{i}$-adapted processes.

We will first show, in some simple examples, that we must expect neither

$$
\tilde{\tau}^{(N)}(i) \rightarrow \tilde{\tau}(i) \quad \text { in probability }
$$

nor

$$
Y\left(0 ; \tilde{\tau}^{(N)}\right) \rightarrow Y(0 ; \tilde{\tau})
$$

when

$$
\lim _{N \rightarrow \infty} \varepsilon^{(N)}(i)=0, \quad \text { P-almost surely (P-a.s.). }
$$

Example 4.1. (i) Suppose that $\left(\xi_{N}\right)_{N \in \mathbb{N}}$ is a sequence of independent binary trials with $\mathrm{P}\left(\xi_{N}=\right.$ $1)=\mathrm{P}\left(\xi_{N}=0\right)=\frac{1}{2}$. We define the process $(Z(i): i=0,1)$ by $Z(0)=Z(1):=1$. The $\sigma$-field $\mathscr{F}_{0}=\mathscr{F}_{1}$ is that generated by the sequence of trials. Moreover, the sequence of perturbations is defined by $\varepsilon^{(N)}(0):=\xi_{N} / N$ and $\varepsilon^{(N)}(1):=0$. Then, starting with any consistent stopping family $\tau$, we obtain $\tilde{\tau}^{(N)}(0)=\xi_{N}$. In particular, no subsequence of $\tilde{\tau}^{(N)}(0)$ converges in probability.

(ii) Let $\Omega=\left\{\omega_{0}, \omega_{1}\right\}$, let $\mathcal{F}$ be the power set of $\Omega$, and let $\mathrm{P}\left(\left\{\omega_{1}\right\}\right)=\frac{1}{4}=1-\mathrm{P}\left(\left\{\omega_{0}\right\}\right)$. We define the process $(Z(i): i=0,1,2)$ by

$$
Z(0)=Z(2):=2, \quad Z\left(1, \omega_{0}\right):=1, \quad Z\left(1, \omega_{1}\right):=3,
$$

and let $\mathcal{F}_{i}$ denote the filtration generated by $Z$. We start with the stopping family $\tau(i)=i$. As $\mathrm{E}[Z(1)]=\frac{3}{2}$, we have

$$
Z(0)=2 \geq \max \left\{\frac{3}{2}, 2\right\}=\max \{\mathrm{E}[Z(1)], \mathrm{E}[Z(2)]\}=\hat{Y}(0, \tau) .
$$

Therefore, $\tilde{\tau}(0)=0$ and $Y(0 ; \tilde{\tau})=2$. The perturbation sequence $\varepsilon^{(N)}$ is defined to be $\varepsilon^{(N)}(1)=$ $\varepsilon^{(N)}(2):=0$ and $\varepsilon^{(N)}(0):=1 / N$. A straightforward calculation shows that, for $N \geq 2$,

$$
\tilde{\tau}^{(N)}\left(0, \omega_{0}\right)=2, \quad \tilde{\tau}^{(N)}\left(0, \omega_{1}\right)=1 .
$$

Thus,

$$
Y\left(0 ; \tilde{\tau}^{(N)}\right)=\frac{9}{4}>2=Y(0 ; \tilde{\tau})
$$

which violates (4.1). 
At first glance, Example 4.1 paints a rather sceptical picture of the stability properties of the one-step improvement. Indeed, the best we can now hope for is that

(ia) there is a sequence, $\bar{\tau}^{(N)}$, of improvers of $\tau$ such that

$$
\left|\tilde{\tau}^{(N)}(i)-\bar{\tau}^{(N)}(i)\right| \rightarrow 0 \quad \text { P-a.s. }
$$

and

(iia) the shortfall of $Y\left(i ; \tilde{\tau}^{(N)}\right)$ below $Y(i ; \tilde{\tau})$ converges to 0 P-a.s.

Note, however, that convergence of the shortfall as in (iia) is the relevant question, not convergence of the distance as in (4.1), since the shortfall corresponds to a change for the worse of $\tilde{\tau}^{(N)}$ compared to $\tilde{\tau}$. As we are interested in an improvement, it suffices to guarantee that such a change for the worse converges to 0 . An additional improvement of $\tilde{\tau}^{(N)}$ compared to $\tilde{\tau}$ due to the error processes $\varepsilon^{(N)}$ may be seen as a welcome side effect.

We now prove assertions (ia) and (iia). We first introduce a new sequence of stopping families which turns out to consist of improvers. Let us define $\bar{\tau}^{(N)}(k)=k$ and, for $1 \leq i<k$, with ' $\vee$ ' denoting logical 'or',

$$
\begin{array}{lll}
\bar{\tau}^{(N)}(i)=i & \Longleftrightarrow \quad\left(\tilde{\tau}^{(M)}(i)>i \text { for only finitely many } M\right) \\
& \vee\left(\tilde{\tau}^{(M)}(i)=i \text { for infinitely many } M \text { and } \tilde{\tau}^{(N)}(i)=i\right), \\
\bar{\tau}^{(N)}(i) \neq i \quad & \bar{\tau}^{(N)}(i)=\bar{\tau}^{(N)}(i+1) .
\end{array}
$$

We then have the following result.

Theorem 4.1. Suppose that

$$
\lim _{N \rightarrow \infty} \varepsilon^{(N)}(i)=0 \quad \text { P-a.s. }
$$

for all $i, 0 \leq i \leq k$. Then $\bar{\tau}^{(N)}$ is an improver of $\tau$ for every $N \in \mathbb{N}$.

Proof. The consistency property (3.1) is satisfied by definition. We show (3.12) by backward induction on $i$. The case $i=k$ is immediate. Suppose that $0 \leq i \leq k-1$ and that (3.12) holds for $i+1$. On $\left\{\tilde{\tau}^{(M)}(i)=i\right.$ for infinitely many $\left.M\right\}$ we have, for infinitely many $M$ (depending on $\omega), Z(i) \geq \hat{Y}(i ; \tau)+\varepsilon^{(M)}(i)$. This means that

$$
Z(i) \geq \hat{Y}(i ; \tau) \quad \text { on }\left\{\tilde{\tau}^{(M)}(i)=i \text { for infinitely many } M\right\}
$$

as $\varepsilon^{(M)}(i)$ tends to 0 , almost surely. However, on the one hand,

$$
\left\{\bar{\tau}^{(N)}(i)=i\right\} \subset\left\{\tilde{\tau}^{(M)}(i)=i \text { for infinitely many } M\right\} .
$$

Thus,

$$
Z(i) \geq \hat{Y}(i ; \tau) \quad \text { on }\left\{\bar{\tau}^{(N)}(i)=i\right\}
$$

but this implies that $\tilde{\tau}(i)=i$ on $\left\{\bar{\tau}^{(N)}(i)=i\right\}$. Consequently, (3.12) holds on $\left\{\bar{\tau}^{(N)}(i)=i\right\}$. 
On the other hand,

$$
\left\{\bar{\tau}^{(N)}(i)>i\right\} \subset\left\{\tilde{\tau}^{(M)}(i)>i \text { for infinitely many } M\right\}
$$

and an analogous argument yields

$$
Z(i) \leq \hat{Y}(i ; \tau) \quad \text { on }\left\{\bar{\tau}^{(N)}(i)>i\right\} .
$$

Consequently $\hat{\tau}(i)>i$ and, thus, by the induction hypothesis,

$$
\bar{\tau}^{(N)}(i)=\bar{\tau}^{(N)}(i+1) \leq \hat{\tau}(i+1)=\hat{\tau}(i) \quad \text { on }\left\{\bar{\tau}^{(N)}(i)>i\right\} .
$$

The induction hypothesis can be applied in the same way to show that

$$
\bar{\tau}^{(N)}(i) \geq \tilde{\tau}(i) \quad \text { on }\left\{\bar{\tau}^{(N)}(i)>i\right\} \cap\{\tilde{\tau}(i)>i\},
$$

an inequality that is trivially satisfied on $\left\{\bar{\tau}^{(N)}(i)>i\right\} \cap\{\tilde{\tau}(i)=i\}$. This completes the proof of (3.12).

The next theorem completes the proof of assertion (ia).

Theorem 4.2. We have

$$
\left|\tilde{\tau}^{(N)}(i)-\bar{\tau}^{(N)}(i)\right| \rightarrow 0 \quad \text { P-a.s. }
$$

or, equivalently,

$$
\mathrm{P}\left(\bigcap_{N \in \mathbb{N}} \bigcup_{M=N}^{\infty}\left\{\tilde{\tau}^{(M)}(i) \neq \bar{\tau}^{(M)}(i)\right\}\right)=0 .
$$

Proof. The statement is obvious for $i=k$. Suppose that $0 \leq i \leq k-1$ and that the statement holds for $i+1$. Define

$$
A(N, i)=\bigcup_{M=N}^{\infty}\left\{\tilde{\tau}^{(M)}(i) \neq \bar{\tau}^{(M)}(i)\right\}
$$

Clearly

$$
A(N, i)=B(N, i) \cup C(N, i) \cup D(N, i),
$$

where

$$
\begin{aligned}
& B(N, i)=\bigcup_{M=N}^{\infty}\left\{\tilde{\tau}^{(M)}(i)=i\right\} \cap\left\{\bar{\tau}^{(M)}(i)>i\right\}, \\
& C(N, i)=\bigcup_{M=N}^{\infty}\left\{\tilde{\tau}^{(M)}(i)>i\right\} \cap\left\{\bar{\tau}^{(M)}(i)=i\right\}, \\
& D(N, i)=\bigcup_{M=N}^{\infty}\left\{\tilde{\tau}^{(M)}(i)>i\right\} \cap\left\{\bar{\tau}^{(M)}(i)>i\right\} \cap\left\{\tilde{\tau}^{(M)}(i) \neq \bar{\tau}^{(M)}(i)\right\} .
\end{aligned}
$$

Since the sets $B(N, i), C(N, i)$, and $D(N, i)$ are decreasing in $N$, we have

$$
\bigcap_{N \in \mathbb{N}} A(N, i)=\left(\bigcap_{N \in \mathbb{N}} B(N, i)\right) \cup\left(\bigcap_{N \in \mathbb{N}} C(N, i)\right) \cup\left(\bigcap_{N \in \mathbb{N}} D(N, i)\right) .
$$


We will show that the three sets on the right-hand side are evanescent. First, as $\bar{\tau}^{(M)}$ and $\tilde{\tau}^{(M)}$ are consistent, it holds that $D(N, i) \subset A(N, i+1)$. Hence, the intersection of the $D(N, i)$ is a null set by the induction hypothesis. By the definition of $\bar{\tau}^{(M)}$, we have

$$
C(N, i) \subset \bigcup_{M=N}^{\infty}\left\{\tilde{\tau}^{(M)}(i)>i\right\} \cap\left\{\tilde{\tau}^{(K)}(i)>i \text { for only finitely many } K\right\} .
$$

Thus, the intersection of the $C(N, i)$ is a null set. A similar argument applies to the intersection of the $B(N, i)$.

Assertion (iia) follows from the next theorem.

Theorem 4.3. Suppose that, for all $i, 0 \leq i \leq k$,

$$
\lim _{N \rightarrow \infty} \varepsilon^{(N)}(i)=0 \quad \text { P-a.s. }
$$

Then, for all $i, 0 \leq i \leq k$, we have

$$
\lim _{N \rightarrow \infty}\left|Y\left(i ; \tilde{\tau}^{(N)}\right)-Y\left(i ; \bar{\tau}^{(N)}\right)\right|=0 \quad \text { P-a.s. }
$$

and

$$
\lim _{N \rightarrow \infty}\left(Y\left(i ; \tilde{\tau}^{(N)}\right)-Y(i ; \tilde{\tau})\right)_{-}=0 \quad \text { P-a.s. }
$$

Remark 4.1. By the dominated convergence theorem, the above convergences also hold in $L^{1}(\mathrm{P})$, the space of $\mathrm{P}$-integrable random variables.

Proof of Theorem 4.3. The first claim is easily derived from Theorem 4.2 and dominated convergence. The second then follows from Proposition 3.1 and Theorem 4.1.

Remark 4.2. Applying the left-hand inequality of Proposition 3.1 yields

$$
\lim _{N \rightarrow \infty}\left(Y\left(i ; \tilde{\tau}^{(N)}\right)-Y(i ; \hat{\tau})\right)_{+}=0 \quad \text { P-a.s. }
$$

Thus, convergence of $Y\left(i ; \tilde{\tau}^{(N)}\right)$ to $Y(i ; \tilde{\tau})$ holds whenever $\tilde{\tau}(i)=\hat{\tau}(i)$ for all $i, 0 \leq i \leq k$.

\subsection{Stability of the algorithm: the case $L=1$}

We are now going to explain how the stability result for the one-step improvement carries over to the algorithm in the case of one exercise right. We will make use of the following perturbed monotonicity result.

Proposition 4.1. Suppose that $\left(\tau_{N}\right)$ is a sequence of consistent stopping families and that, for all $i, 0 \leq i \leq k$,

$$
\lim _{N \rightarrow \infty}\left(Y\left(i ; \tau_{N}\right)-Y(i ; \tau)\right)_{-}=0 \quad \text { P-a.s. }
$$

Then, for all $i, 0 \leq i \leq k$,

$$
\lim _{N \rightarrow \infty}\left(Y\left(i ; \tilde{\tau}_{N}\right)-Y(i ; \tilde{\tau})\right)_{-}=0 \quad \text { P-a.s. },
$$

where

$$
\tilde{\tau}_{N}(i):=\inf \left\{j: i \leq j \leq k, \hat{Y}\left(j ; \tau_{N}\right) \leq Z(j)\right\}
$$


Remark 4.3. For a sequence such that $\tau_{N}=\sigma$ for all $N$, with $\sigma$ being consistent, Proposition 4.1 states that

$$
Y(i ; \sigma) \geq Y(i ; \tau) \quad \Longrightarrow \quad Y(i ; \tilde{\sigma}) \geq Y(i ; \tilde{\tau}) .
$$

Hence, the better the input stopping family, the better the improvement.

Proof of Proposition 4.1. The statement will be proved by backward induction on $i$. The induction base (i.e. the result for $i=k$ ) is obvious. Suppose that the statement holds for some $i+1,1 \leq i+1 \leq k$.

We first note that, by Remark 3.2,

$$
\mathbf{1}_{\{\tilde{\tau}(i)=i\}}\left(Y\left(i ; \tilde{\tau}_{N}\right)-Y(i ; \tilde{\tau})\right)_{-} \leq\left(Y\left(i ; \tilde{\tau}_{N}\right)-Z(i)\right)_{-}=0 .
$$

We next show that the statement is true on the set $\left\{\tilde{\tau}_{M}(i)=i\right.$ for infinitely many $\left.M\right\}$. For this we need the following preliminary result. By Jensen's inequality and the dominated convergence theorem, for all $p \geq i$ we have

$$
\left(\mathrm{E}^{\mathcal{F}_{i}}\left[Y\left(p ; \tau_{N}\right)\right]-\mathrm{E}^{\mathcal{F}_{i}}[Y(p ; \tau)]\right)_{-} \leq \mathrm{E}^{\mathcal{F}_{i}}\left[\left(Y\left(p ; \tau_{N}\right)-Y(p ; \tau)\right)_{-}\right] \rightarrow 0 .
$$

Thus,

$$
\lim _{N \rightarrow \infty}\left(\hat{Y}\left(i ; \tau_{N}\right)-\hat{Y}(i ; \tau)\right)_{-}=0 \quad \text { P-a.s., }
$$

since the operator ' $m a x$ ' is continuous with respect to the metric generated by the negative part. On $\left\{\tilde{\tau}_{M}(i)=i\right.$ for infinitely many $\left.M\right\}$ we have, for infinitely many $M, \hat{Y}\left(i ; \tau_{M}\right) \leq Z(i)$. Since

$$
(Z(i)-\hat{Y}(i ; \tau))_{-} \leq\left(Z(i)-\hat{Y}\left(i ; \tau_{M}\right)\right)_{-}+\left(\hat{Y}\left(i ; \tau_{M}\right)-\hat{Y}(i ; \tau)\right)_{-},
$$

we may conclude from (4.3) that

$$
Z(i) \geq \hat{Y}(i ; \tau) \quad \text { on }\left\{\tilde{\tau}_{M}(i)=i \text { for infinitely many } M\right\} .
$$

Hence,

$$
\left\{\tilde{\tau}_{M}(i)=i \text { for infinitely many } M\right\} \subset\{\tilde{\tau}(i)=i\} .
$$

On the latter set the statement was proved in (4.2).

It remains to verify the statement on the set

$$
E(i)=\left\{\tilde{\tau}_{M}(i)=i \text { for only finitely many } M\right\} \cap\{\tilde{\tau}(i)>i\} .
$$

Define

$$
N_{0}(i)=\mathbf{1}_{E(i)} \max \left\{N: \tilde{\tau}_{N}(i)=i\right\}+1,
$$

and note that the process $N_{0}(i)$ is $\mathcal{F}_{i}$-adapted. Since

$$
\tilde{\tau}_{N}(i)>i \quad \text { on }\left\{N \geq N_{0}(i)\right\} \cap E(i),
$$

it follows from the consistency of $\tilde{\tau}_{N}$, the induction hypothesis, Jensen's inequality, and the dominated convergence theorem that

$$
\begin{aligned}
\mathbf{1}_{\{N \geq} & \left.N_{0}(i)\right\} \cap E(i) \\
& =\mathbf{1}_{\left\{N \geq N_{0}(i)\right\} \cap E(i)}\left(\mathrm{E}^{\mathcal{F}_{i}}\left[Y\left(i+1 ; \tilde{\tau}_{N}\right)\right]-\mathrm{E}^{\mathcal{F}_{i}}[Y(i+1 ; \tilde{\tau})]\right)_{-} \\
& \leq \mathrm{E}^{\mathcal{F}_{i}}\left[\left(Y\left(i+1 ; \tilde{\tau}_{N}\right)-Y(i+1 ; \tilde{\tau})\right)_{-}\right] \\
& \rightarrow 0 .
\end{aligned}
$$

This completes the proof. 
For notational convenience we state the stability result of the algorithm for two improvement steps $(m=2)$ only. It is immediately obvious how this extends to higher iterations. We will also omit all subscripts, which are superfluous in the case of one exercise right. For instance, we write $\tau^{(1)}$ instead of $\tau_{1,1}^{(1)}$. First note that, with $\tau=\tau^{(0)}$,

$$
\begin{aligned}
\tau^{(1)}(i) & =\tilde{\tau}(i), \\
\tau^{(2)}(i) & =\tilde{\tilde{\tau}}(i):=\inf \{j: i \leq j \leq k, \hat{Y}(j ; \tilde{\tau}) \leq Z(j)\} .
\end{aligned}
$$

Let us suppose that, for $\left(N_{1}, N_{2}\right) \in \mathbb{N} \times \mathbb{N}$, we are given sequences $\varepsilon^{\left(N_{1}\right)}(i)$ and $\varepsilon^{\left(N_{1}, N_{2}\right)}(i)$ such that

$$
\lim _{N_{1} \rightarrow \infty} \varepsilon^{\left(N_{1}\right)}(i)=0 \quad \text { P-a.s. }
$$

for $0 \leq i \leq k$ and

$$
\lim _{N_{2} \rightarrow \infty} \varepsilon^{\left(N_{1}, N_{2}\right)}(i)=0 \quad \text { P-a.s. }
$$

for $0 \leq i \leq k$ and $N_{1} \in \mathbb{N}$. We then define

$$
\begin{aligned}
\tilde{\tau}^{\left(N_{1}\right)}(i) & :=\inf \left\{j: i \leq j \leq k, \hat{Y}(j ; \tau)+\varepsilon^{\left(N_{1}\right)}(j) \leq Z(j)\right\} \quad \text { (as above), } \\
\tilde{\tau}^{\left(N_{1}\right)}(i) & :=\inf \left\{j: i \leq j \leq k, \hat{Y}\left(j ; \tilde{\tau}^{\left(N_{1}\right)}\right) \leq Z(j)\right\}, \\
\tilde{\tau}^{\left(N_{1}, N_{2}\right)}(i) & :=\inf \left\{j: i \leq j \leq k, \hat{Y}\left(j ; \tilde{\tau}^{\left(N_{1}\right)}\right)+\varepsilon^{\left(N_{1}, N_{2}\right)}(j) \leq Z(j)\right\} .
\end{aligned}
$$

Theorem 4.3 now yields

$$
\begin{aligned}
\lim _{N_{1} \rightarrow \infty}\left(Y\left(i ; \tilde{\tau}^{\left(N_{1}\right)}\right)-Y(i ; \tilde{\tau})\right)_{-}=0 & \text { P-a.s. } \\
\lim _{N_{2} \rightarrow \infty}\left(Y\left(i ; \tilde{\tilde{\tau}}^{\left(N_{1}, N_{2}\right)}\right)-Y\left(i ; \tilde{\tilde{\tau}}^{\left(N_{1}\right)}\right)\right)_{-}=0 & \text { P-a.s. }
\end{aligned}
$$

In view of (4.4) we obtain, from Proposition 4.1,

$$
\lim _{N_{1} \rightarrow \infty}\left(Y\left(i ; \tilde{\tilde{\tau}}^{\left(N_{1}\right)}\right)-Y(i ; \tilde{\tilde{\tau}})\right)_{-}=0 \quad \text { P-a.s. }
$$

Since

$$
\left(Y\left(i ; \tilde{\tilde{\tau}}^{\left(N_{1}, N_{2}\right)}\right)-Y^{(2)}(i)\right)_{-} \leq\left(Y\left(i ; \tilde{\tilde{\tau}}^{\left(N_{1}, N_{2}\right)}\right)-Y\left(i ; \tilde{\tilde{\tau}}^{\left(N_{1}\right)}\right)\right)_{-}+\left(Y\left(i ; \tilde{\tilde{\tau}}^{\left(N_{1}\right)}\right)-Y(i ; \tilde{\tilde{\tau}})\right)_{-},
$$

we then obtain the following theorem.

Theorem 4.4. For all $i, 0 \leq i \leq k$,

$$
\lim _{N_{1} \rightarrow \infty} \lim _{N_{2} \rightarrow \infty}\left(Y\left(i ; \tilde{\tilde{\tau}}^{\left(N_{1}, N_{2}\right)}\right)-Y^{(2)}(i)\right)_{-}=0 \quad \text { P-a.s. }
$$

and in $L^{1}(\mathrm{P})$.

The generalization of this result to $m$ iteration steps may be put into words as follows: the shortfall of the investor's expected gain corresponding to $m$ perturbed steps of the algorithm below the expected gain corresponding to $m$ theoretical steps converges to 0 . 


\subsection{Stability under several exercise rights}

The stability issue becomes more involved under several exercise rights. One reason is that we cannot expect the inequality

$$
Y_{L}\left(i ; \bar{\sigma}_{1}, \ldots, \bar{\sigma}_{L}\right) \geq Y_{L}\left(i ; \tilde{\sigma}_{1}, \ldots, \tilde{\sigma}_{L}\right),
$$

where $\bar{\sigma}_{1}, \ldots, \bar{\sigma}_{L}$ are arbitrary improvers of $\sigma_{1}, \ldots, \sigma_{L}$, to hold, but rather only the inequalities stated in Theorem 3.5. That theorem suggests that we must satisfy ourselves with the following stability result for the one-step improvement under several exercise rights.

Theorem 4.5. Suppose that $\sigma_{1}, \ldots, \sigma_{D}$ are consistent stopping families. For $1 \leq L \leq D$, define

$$
\begin{aligned}
\tilde{\sigma}_{L}^{(N)}(i)=\inf \left\{j: j \geq i, Z(j)+\mathrm{E}^{\mathcal{F}_{j}}\left[Y_{L-1}\left(j+\delta ; \sigma_{1}, \ldots, \sigma_{L-1}\right)\right]\right. & \\
& \left.\geq \hat{Y}_{L}\left(j ; \sigma_{1}, \ldots, \sigma_{L}\right)+\varepsilon_{L}^{(N)}(j)\right\},
\end{aligned}
$$

where, for all $L, 1 \leq L \leq D$, and $i, 0 \leq i \leq k$,

$$
\lim _{N \rightarrow \infty} \varepsilon_{L}^{(N)}(i)=0 \quad \text { P-a.s. }
$$

Then there are sequences of improvers, $\bar{\sigma}_{1}^{(N)}, \ldots, \bar{\sigma}_{D}^{(N)}$, of $\sigma_{1}, \ldots, \sigma_{D}$ such that, for all $L, 1 \leq$ $L \leq D$,

$$
\lim _{N \rightarrow \infty}\left|\tilde{\sigma}_{L}^{(N)}(i)-\bar{\sigma}_{L}^{(N)}(i)\right|=0
$$

Moreover,

$$
\lim _{N \rightarrow \infty}\left|Y_{L}\left(i ; \tilde{\sigma}_{1}^{(N)}, \ldots, \tilde{\sigma}_{L}^{(N)}\right)-Y_{L}\left(i ; \bar{\sigma}_{1}^{(N)}, \ldots, \bar{\sigma}_{L}^{(N)}\right)\right|=0 \quad \text { P-a.s. }
$$

and

$$
\lim _{N \rightarrow \infty}\left(Y_{L}\left(i ; \tilde{\sigma}_{1}^{(N)}, \ldots, \tilde{\sigma}_{L}^{(N)}\right)-Y_{L}\left(i ; \sigma_{1}, \ldots, \sigma_{L-1}, \tilde{\sigma}_{L}\right)\right)_{-}=0 \quad \text { P-a.s. }
$$

Proof. In view of Lemma 3.2 and Theorem 3.5, the theorem follows by straightforward reduction to the case of one exercise right.

A more satisfactory result can be derived for the $y_{L}$-algorithm (i.e. (3.8)) under an additional assumption.

Theorem 4.6. Under the assumptions (and with the notation) of the previous theorem, suppose additionally that, for $1 \leq i \leq k-1$ and $1 \leq L \leq D$,

$$
\mathrm{P}\left(Z_{L}\left(i ; \sigma_{1}, \ldots, \sigma_{L-1}\right)=\max _{p \geq i+1} \mathrm{E}^{\mathcal{F}_{i}}\left[Z_{L}\left(\sigma_{L}(p) ; \sigma_{1}, \ldots, \sigma_{L-1}\right)\right]\right)=0 .
$$

Then, for $0 \leq i \leq k$ and $1 \leq L \leq D$,

$$
\lim _{N \rightarrow \infty}\left|y_{L}\left(i ; \tilde{\sigma}_{1}^{(N)}, \ldots, \tilde{\sigma}_{L}^{(N)}\right)-y_{L}\left(i ; \tilde{\sigma}_{1}, \ldots, \tilde{\sigma}_{L}\right)\right|=0 \quad \mathrm{P} \text {-a.s. }
$$

Proof. Assumption (4.5) guarantees that $\tilde{\sigma}_{L}(i)=\hat{\sigma}_{L}(i)$ for $1 \leq i \leq k$ and $1 \leq L \leq D$. Thus, $\bar{\sigma}_{L}^{(N)}(i)=\tilde{\sigma}_{L}(i)$ for $1 \leq i \leq k, 1 \leq L \leq D$, and $N \in \mathbb{N}$, by the definition of an 
improver. (Here $\bar{\sigma}_{L}^{(N)}(i)$ denotes the sequences introduced in Theorem 4.5.) For $1 \leq i \leq k$ and $1 \leq L \leq D$, Theorem 4.5 now yields

$$
\lim _{N \rightarrow \infty} \tilde{\sigma}_{L}^{(N)}(i)=\tilde{\sigma}_{L}(i) \quad \text { P-a.s. }
$$

Hence, the assertion follows from the definition of $y_{L}$ and an application of the dominated convergence theorem. (Recall that the definition of $y_{L}$ does not involve the value of the stopping families at time 0.)

We now discuss the stability of the $y_{L}$-algorithm. We again demonstrate the stability of the multiple stopping algorithm for only two steps $(m=2)$. Suppose that we are given consistent starting families $\sigma_{1}, \ldots, \sigma_{D}$ (with the superscript ' 0 ' suppressed in the notation of the algorithm). Recall that

$$
\begin{aligned}
\sigma_{L}^{(1)}(i) & :=\tilde{\sigma}_{L}(i), \\
\sigma_{L}^{(2)}(i) & :=\tilde{\sigma}_{L}^{(1)}(i)=\tilde{\tilde{\sigma}}_{L}(i) .
\end{aligned}
$$

We shall suppose that both (4.5) and its analogue for $\tilde{\sigma}_{L}$ hold, i.e. that, for $1 \leq i \leq k-1$ and $1 \leq L \leq D$

$$
\mathrm{P}\left(Z_{L}\left(i ; \tilde{\sigma}_{1}, \ldots, \tilde{\sigma}_{L-1}\right)=\max _{p \geq i+1} \mathrm{E}^{\mathcal{F}_{i}}\left[Z_{L}\left(\tilde{\sigma}_{L}(p) ; \tilde{\sigma}_{1}, \ldots, \tilde{\sigma}_{L-1}\right)\right]\right)=0 .
$$

We next consider the perturbed versions,

$$
\begin{gathered}
\tilde{\sigma}_{L}^{\left(N_{1}\right)}(i)=\inf \left\{j: j \geq i, Z(j)+\mathrm{E}^{\mathcal{F}_{j}}\left[Y_{L-1}\left(j+\delta ; \sigma_{1}, \ldots, \sigma_{L-1}\right)\right]\right. \\
\left.\geq \hat{Y}_{L}\left(j ; \sigma_{1}, \ldots, \sigma_{L}\right)+\varepsilon_{L}^{\left(N_{1}\right)}(j)\right\}, \\
\tilde{\tilde{\sigma}}_{L}^{\left(N_{1}, N_{2}\right)}(i)=\inf \left\{j: j \geq i, Z(j)+\mathrm{E}^{\mathcal{F}_{j}}\left[Y_{L-1}\left(j+\delta ; \tilde{\sigma}_{1}^{\left(N_{1}\right)}, \ldots, \tilde{\sigma}_{L-1}^{\left(N_{1}\right)}\right)\right]\right. \\
\left.\geq \hat{Y}_{L}\left(j ; \tilde{\sigma}_{1}^{\left(N_{1}\right)}, \ldots, \tilde{\sigma}_{L}^{\left(N_{1}\right)}\right)+\varepsilon_{L}^{\left(N_{1}, N_{2}\right)}(j)\right\},
\end{gathered}
$$

with

$$
\begin{aligned}
\lim _{N_{1} \rightarrow \infty} \varepsilon_{L}^{\left(N_{1}\right)}(i)=0 & \text { P-a.s., } \\
\lim _{N_{2} \rightarrow \infty} \varepsilon_{L}^{\left(N_{1}, N_{2}\right)}(i)=0 & \text { P-a.s. }
\end{aligned}
$$

We denote by $\tilde{\tilde{\sigma}}_{L}^{\left(N_{1}\right)}$ the theoretical improvement of $\tilde{\sigma}_{L}^{\left(N_{1}\right)}$ under the tilde operation. The additional assumption (4.5) now ensures that, for $1 \leq i \leq k$,

$$
\lim _{N \rightarrow \infty} \tilde{\sigma}_{L}^{(N)}(i)=\tilde{\sigma}_{L}(i) \quad \text { P-a.s. }
$$

(see the proof of Theorem 4.6). Thus, we can write (applying Lemma 3.2),

$$
\begin{aligned}
\tilde{\tilde{\sigma}}_{L}^{\left(N_{1}\right)}(i)=\inf \left\{j: j \geq i, Z_{L}\left(j ; \tilde{\sigma}_{1}, \ldots, \tilde{\sigma}_{L-1}\right)\right. & \\
& \left.\geq \max _{p \geq j+1} \mathrm{E}^{\mathcal{F}_{j}}\left[Z_{L}\left(\tilde{\sigma}_{L}(p) ; \tilde{\sigma}_{1}, \ldots, \tilde{\sigma}_{L-1}\right)\right]+\tilde{\varepsilon}_{L}^{\left(N_{1}\right)}(i)\right\},
\end{aligned}
$$

where

$$
\lim _{N_{1} \rightarrow \infty} \tilde{\varepsilon}_{L}^{\left(N_{1}\right)}(i)=0 \quad \text { P-a.s. }
$$


We now define $\overline{\tilde{\sigma}}_{L}^{\left(N_{1}\right)}(k)=k$ and, for $1 \leq i<k$,

$$
\begin{array}{lll}
\overline{\tilde{\sigma}}_{L}^{\left(N_{1}\right)}(i)=i & \Longleftrightarrow \quad\left(\tilde{\tilde{\sigma}}_{L}^{(M)}(i)>i \text { for only finitely many } M\right) \\
& \vee\left(\tilde{\tilde{\sigma}}_{L}^{(M)}(i)=i \text { for infinitely many } M \text { and } \tilde{\tilde{\sigma}}_{L}^{\left(N_{1}\right)}(i)=i\right), \\
\overline{\tilde{\sigma}}_{L}^{\left(N_{1}\right)}(i) \neq i \quad \Longrightarrow & \overline{\tilde{\sigma}}_{L}^{\left(N_{1}\right)}(i)=\overline{\tilde{\sigma}}_{L}^{\left(N_{1}\right)}(i+1) .
\end{array}
$$

By Theorem 4.2, for all $i, 1 \leq i \leq k$ and $L, 1 \leq L \leq D$, we have

$$
\lim _{N_{1} \rightarrow \infty}\left|\tilde{\tilde{\sigma}}_{L}^{\left(N_{1}\right)}(i)-\overline{\tilde{\sigma}}_{L}^{\left(N_{1}\right)}(i)\right|=0 .
$$

However, assumption (4.6) implies that the improvers $\overline{\tilde{\sigma}}_{L}^{\left(N_{1}\right)}(i)$ coincide with $\tilde{\tilde{\sigma}}_{L}(i)$ for $1 \leq i \leq$ $k$. Hence, the dominated convergence theorem yields, on the one hand,

$$
\lim _{N_{1} \rightarrow \infty}\left|y_{L}\left(i ; \tilde{\tilde{\sigma}}_{1}^{\left(N_{1}\right)}, \ldots, \tilde{\tilde{\sigma}}_{L}^{\left(N_{1}\right)}\right)-y_{L}\left(i ; \tilde{\tilde{\sigma}}_{1}, \ldots \tilde{\tilde{\sigma}}_{L}\right)\right|=0 \quad \text { P-a.s. }
$$

On the other hand, a direct application of Theorem 4.6 gives

$$
\lim _{N_{2} \rightarrow \infty}\left|y_{L}\left(i ; \tilde{\tilde{\sigma}}_{1}^{\left(N_{1}, N_{2}\right)}, \ldots, \tilde{\tilde{\sigma}}_{L}^{\left(N_{1}, N_{2}\right)}\right)-y_{L}\left(i ; \tilde{\tilde{\sigma}}_{1}^{\left(N_{1}\right)}, \ldots \tilde{\tilde{\sigma}}_{L}^{\left(N_{1}\right)}\right)\right|=0 \quad \text { P-a.s. }
$$

The discussion is summarized in the following theorem.

Theorem 4.7. Suppose that (4.5) and (4.6) hold. Then, for all $i, 0 \leq i \leq k$ and $L, 1 \leq L \leq D$,

$$
\lim _{N_{1} \rightarrow \infty} \lim _{N_{2} \rightarrow \infty}\left|y_{L}\left(i ; \tilde{\tilde{\sigma}}_{1}^{\left(N_{1}, N_{2}\right)}, \ldots, \tilde{\tilde{\sigma}}_{L}^{\left(N_{1}, N_{2}\right)}\right)-y_{L}^{(2)}(i)\right|=0 \quad \text { P-a.s. }
$$

and in $L^{1}(\mathrm{P})$.

The straightforward generalization to higher-order iterations is left to the reader.

Remark 4.4. (i) Stability of the $Y_{L}$-algorithm can be proven along the same lines if (4.5) and (4.6) also hold for $i=0$. We emphasize, however, that this additional assumption can always be violated by a poor choice of the constant $Z(0)$. This is why we refrained from making this additional assumption and presented the stability analysis for the $y_{L}$-algorithm.

(ii) Assumptions (4.5) and (4.6) can be replaced by the weaker condition that the limits

$$
\lim _{N_{1} \rightarrow \infty} \tilde{\sigma}_{L}^{\left(N_{1}\right)}(i), \quad \lim _{N_{1} \rightarrow \infty} \lim _{N_{2} \rightarrow \infty} \tilde{\tilde{\sigma}}_{L}^{\left(N_{1}, N_{2}\right)}(i)
$$

exist for $1 \leq i \leq k-1$ and $1 \leq L \leq D$. Then $y_{L}^{(2)}(i)$ in Theorem 4.7 must be replaced by some theoretical $y_{L}$-two-step improvement of $\left(\sigma_{1}, \ldots, \sigma_{l}\right)$. An analogous result holds for the $Y_{L}$-algorithm when the limits also exist for $i=0$.

\section{Acknowledgement}

We are very grateful to Anastasia Kolodko for an important comment that helped to strengthen Theorem 3.1, concerning the number of iterations needed for termination. 


\section{References}

ANDERSEN, L. (1999). A simple approach to the pricing of Bermudan swaptions in the multifactor LIBOR market model. J. Comput. Finance 3, 5-32.

Bender, C. AND Schoenmakers, J. (2006). Addendum to 'An iterative method for multiple stopping: convergence and stability'. Available at http://www.wias-berlin.de/people/schoenma/.

BertseKas, D. P. (2001). Dynamic Programming and Optimal Control, Vol. II, 2nd edn. Athena Scientific, Belmont, MA.

Broadie, M. ANd Glasserman, P. (2004). A stochastic mesh method for pricing high-dimensional American options. J. Comput. Finance 7, 35-72.

Broadie, M., Glasserman, P. and Ha, Z. (2000). Pricing American options by simulations using a stochastic mesh with optimized weights. In Probabilistic Constrained Optimization: Methodology and Applications, ed. S. Uryasev, Kluwer, Dordrecht, pp. 26-44.

Carmona, R. And TouzI, N. (2006). Optimal multiple-stopping and valuation of swing options. To appear in Math. Finance.

Haugh, M. B. And Kogan, L. (2004). Pricing American options: a duality approach. Operat. Res. 52, 258-270.

IBÁÑEZ, A. (2004). Valuation by simulation of contingent claims with multiple exercise opportunities. Math. Finance 14, 223-248.

IbáÑEZ, A. AND Zapatero, F. (2004). Monte Carlo valuation of American options through computation of the optimal exercise frontier. J. Financial Quant. Anal. 39, 253-276.

JaIllet, P., Ronn, E. I. AND Tompaidis, S. (2004). Valuation of commodity-based swing options. Manag. Sci. 50, 909-921.

JAMSHIDian, F. (2006). Duality of optimal stopping times and domineering claims: a Doob-Meyer decomposition approach to American options. Working paper.

Kolodko, A. AND Schoenmakers, J. (2006). Iterative construction of the optimal Bermudan stopping time. Finance Stoch. 10, 27-49.

Kushner, H. J. And Dupuis, P. (2001). Numerical Methods for Stochastic Control Problems in Continuous Time, 2nd edn, Springer, New York.

Longstaff, F. A. And Schwartz, E. S. (2001). Valuing American options by simulation: a simple least-square approach. Rev. Financial Stud. 14, 113-147.

Meinshausen, N. And Hambly, B. M. (2004). Monte Carlo methods for the valuation of multiple-exercise options. Math. Finance 14, 557-583.

Milstein, G. N., Reiss, O. And Schoenmakers, J. (2004). A new Monte Carlo method for American options. Internat. J. Theoret. Appl. Finance 7, 591-614.

Neveu, J. (1975). Discrete-Parameter Martingales. North-Holland, Amsterdam.

Puterman, M. L. (1994). Markov Decision Processes: Discrete Stochastic Dynamic Programming. John Wiley, New York.

Rogers, L. C. G. (2002). Monte Carlo valuation of American options. Math. Finance 12, 271-286.

Schoenmakers, J. (2005). Robust Libor Modelling and Pricing of Derivative Products. Chapman and Hall/CRC Press, Boca Raton, FL. 